\section{La Terre, 2108 : un archipel de communautés autonomes}

\author{
FAbrice Flipo
}

Qu'est-ce que l'utopie? L'ouvrage récent de Thierry Paquot fait le point sur le sujet ${ }^{[1]}$. Nous nous appuierons ici sur une interprétation kantienne de l'utopie en tant qu' « idée régulatrice » de la raison. En ce sens, l'utopie est le résultat des conclusions logiques de la raison pratique lorsqu'elles sont mises en œuvre jusqu'au bout de leurs conséquences. La raison pratique n'a pas de lieu concret dans le présent car elle n'a pas de réalité empirique, elle est d'ordre conceptuelle, appelle le donné à être différemment. Mais elle n'est pas non plus dénuée de réalité tout court: l'utopie est l'état du monde vers lequel elle tend, dans ses efforts pour transformer le présent. Elle est ce à quoi aboutit conceptuellement la raison pratique, qui est l'instrument qui permet de séparer le légitime de l'illégitime, le juste de l'injuste. L'utopie, en ce sens, est émancipatrice: elle est ce qui doit avoir lieu dans le monde pour que le règne de la raison et de la justice advienne parmi les êtres humains.

Les utopies auxquelles nous nous intéressons ici sont des utopies transnationales, cosmopolitiques, car les enjeux réunis diplomatiquement sous le terme « développement durable » posent le problème de savoir comment pacifier le genre humain en tant que tel. C'est Kant qui est considéré comme la référence et le précurseur dans ce domaine, au moins en Occident. Pour Kant, la paix perpétuelle et cosmopolitique est la conséquence de l'émergence de la raison pratique universelle ${ }^{[2]}$, enfin sortie de son " état de minorité », c'est-à-dire dégagée de ses préjugés et de l'obscurantisme religieux. C'était là l'un des projets les plus chers des philosophes des Lumières. L'idée kantienne fut largement reprise au $19^{\mathrm{e}}$ siècle. Mais elle fut aussi fortement déformée, notamment par Hegel, Marx et les héritiers d'Adam Smith. La « loi universelle » prit la forme du « développement », dont l'universalité est aujourd'hui questionnée.

Nous nommons «utopie écologiste » un projet dont le maître mot est de conjurer la tragédie des communs exposée par Garrett Hardin comme le productivisme avéré des anciennes économies planifiées. La stratégie principale est d'instituer les services écologiques de res nullius en res communis, comme en témoignent les écrits militants tels
[1] T. Paquot, Utopies et utopistes, La Découverte, Paris, 2007.

[2] E. Kant, Idée d'une histoire universelle du point de vue cosmopolitique,

Gallimard, Paris, 1985 [1784]. Fabrice Flipo est 
que le rapport Europe soutenable des Amis de la Terre Europe ${ }^{[3]}$. Nous considérons ce rapport comme la description la plus aboutie du projet écologiste, car il intègre la quasi-totalité des revendications militantes dans une vision de l'avenir qui tente d'être aussi complète que possible. Nous montrons que cette utopie s'oppose aux utopies cosmopolitiques anciennes, issues du capitalisme ou du socialisme. Nous montrons qu'elle exhibe en outre des filiations avec les Lumières, donc loin d'être un « retour à l'âge de pierre » comme on le dit parfois pour la dénigrer. Peut-on pour autant l'assimiler à un « retour aux Lumières »? C'est la question qui est posée en conclusion.

\section{Les anciennes utopies cosmopolitiques en crise}

Comment justifier l'existence d'une pluralité de peuples ou d'États? Comment éviter qu'ils ne se trouvent dans « l'état de nature », un état dans lequel il n'existe pas de loi positive, risquant ainsi de prolonger indéfiniment une "guerre de tous contre tous », selon le mot de Hobbes? Est-ce uniquement un manque de moyens? Est-ce un stade de l'histoire de l'humanité, appelé à être dépassé ? L'État mondial, ou le Peuple unique, sont-ils la seule manière de mettre fin aux conflits?

La solution proposée par les Lumières est celle proposée par Kant, sous la forme d'un «projet de paix perpétuelle ». Le cosmopolitisme est ancré dans le droit naturel rationnel, c'est-à-dire les droits de l'homme et du citoyen. Il est conçu dans le prolongement du « libéralisme politique », à savoir la séparation de l'Église et de l'État, la limitation des abus politiques par le jeu des contre-pouvoirs et des procédures, et le respect des droits fondamentaux de la personne ${ }^{[4]}$.

Cette solution, élaborée au $18^{\mathrm{e}}$ siècle, évolue bien vite vers une autre proposition, qui se généralise au cours du $19^{\mathrm{e}}$ siècle: le libéralisme économique. Les héritiers d'Adam Smith cherchent à pacifier l'humanité par la lex mercatoria. La division indéfinie du travail et l'inclusion de l'humanité entière dans un même marché mondial sur lequel chacun est libre de louer ses services contre rémunération sont une procédure permettant d'agréger les choix et de générer un bien-être collectif sans

[3] Friends of the Earth Europe, Europe soutenable, 1995, ronéotypé. [4] P. Raynaud, «Libéralisme ", Dictionnaire de philosophie politique, PUF, Paris, 1996. cesse grandissant. La poursuite du profit conduit chacun à s'efforcer d'accroître la productivité et les sociétés voient leur niveau de vie augmenter: l'égoïsme génère des « externalités positives » et se retourne ainsi en bien public. Ce résultat est explicitement visé par les institutions qui sont aujourd'hui en charge de sa réalisation: "International 
trade institutions and liberal trade policies are a means to an end [...] : [the] increase [of] social welfare ${ }^{[5]}$. $\gg$ Cette utopie met les valeurs de travail, production et division du travail au cœur de son projet, et affirme que la rareté est le problème principal. L'insatisfaction savamment entretenue par la publicité et la valorisation du luxe ${ }^{[6]}$ empêchent le système de stagner; dès lors il ne peut que progresser.

Seulement voilà, les marchés sont désormais le lieu de production d'externalités négatives massives, et non plus seulement d'externalités positives : changements climatiques, effondrement de la diversité biologique, etc. Ils sont de plus menacés dans leur existence même par la raréfaction des matières premières et la flambée des prix qui en découlera, générant une exclusion toujours plus importante. Au lieu de la pacification, c'est le conflit qui se fait jour. Que faire?

Sous la plume de Marx, l'utopie technomarchande a déjà fait l'objet d'une critique qui se voulait radicale. L'État et le contrôle démocratique de la production devaient permettre de venir à bout de ces excès des marchés livrés à eux-mêmes, dans l'ignorance qu'ils étaient des buts ultimes de leur propre action. La crise financière actuelle illustre la pertinence et l'actualité de ce rôle nécessaire de l'État. Par exemple, les nationalisations de Fannie Mae et Freddie Mac sont plus qu'une petite entorse à l'utopie technomarchande: elles en remettent en cause le bien-fondé, de manière éclatante. Ce « retour de balancier » est donc aussi un « retour à Marx », qui avait déjà anticipé sur l'irréalisme de la thèse de l'autorégulation des marchés, qui prenait la partie (l'échange marchand) pour le tout (la société). L'État renvoie ainsi à l'existence d'un lien social qui n'est pas basé sur la seule poursuite de l'intérêt financier. Devons-nous alors endosser de nouveau les projets de l'Internationale?

Ce n'est pas évident. En effet, la mondialisation des échanges était perçue par Marx, en dernière analyse, comme une force positive. De concentrations en fusions, le capitalisme, mû par un intérêt étroit dialectiquement en prise avec les âpres luttes ouvrières, produisait peu à peu, à son insu, une socialisation croissante des forces productives, permettant à l'esprit hégélien de retourner progressivement auprès de soi - et à la justice de se produire sur Terre. Le prolétariat, exploité et volé d'une partie de son travail, détourné par des propriétaires peu scrupuleux et imbus d'eux-mêmes, prendrait finalement possession du potentiel technique qu'il aurait suscité. Prendre possession, et non détruire ou modifier. Pour Marx, les forces productives déclenchées par la bourgeoisie sont positives. Elles brisent les attachements locaux ${ }^{[7]}$, qui sont réactionnaires. Il voit dans l'homogénéisation des modes de vie la clé de la coopération planétaire, car elle occasionnera la fin des par-
[5] World Bank, World Development Report 1999-2000, p. 60-61. Notre traduction: "Les institutions du commerce international sont des moyens ordonnés à une seule fin: l'accroissement du bien-être social. "

[6] R. Allard, La querelle sur le luxe au XVIII siècle. Voltaire, Rousseau et la question du bonheur, mémoire de maîtrise, UQAM, Montréal, 2003 (http://agora. qc.ca/thematiques/rousseau. nsf/Theses/La_Querelle_ du_luxe_au_XVIIle_siecle); J. Marseille (dir.), Le luxe en France, ADHE, Paris, 1999; J. Castarède, Histoire du luxe en France, Eyrolles, Paris, 2007.

[7] K. Marx et F. Engels, Le Manifeste du Parti Communiste, Messidor/ Editions Sociales, Paris, 1986 [1844], p. 59.

[8] « Les mêmes modes de production génèrent la même littérature " (K. Marx et F. Engels, op. cit., p. ?). 
[9] A. Negri et M. Hardt, Empire, Exils, Paris, 2000. Voir la critique P. Dardot, M. El Mouhoud et C. Laval,

Sauver Marx? Empire, multitude, travail immatériel, La Découverte, Paris, 2007. [10] Voir F. Flipo, « L'écologie politique est-elle

"réactionnaire" ? L'enjeu des choix technologiques",

Communication pour le Congrès Marx International, 3-6 octobre 2007. [11] J.-M. Vincent, « Critique de l'économisme et économisme chez Marx ", 2008, http://semimarx.free.fr/ article.php3?id_article=202.

[12] Voir la simplicité volontaire, la décroissance, etc.

[13] G. Hardin, «The tragedy of the commons ", Science, $\mathrm{n}^{\circ} 162,1968$, p. $1243-1248$.

[14] Friends of the Earth Europe, op. cit. ticularismes ${ }^{[8]}$. La bourgeoisie est à l'origine, malgré elle, d'un progrès technologique indéniable - thèse que reprend encore Empire d'Antonio Negri et Michael Hardt, en élevant les technologies de l'information au rang de moyen ouvrant la voie au communisme ${ }^{[9]}$.

La critique principale émise par Marx à l'encontre du système capitaliste, à savoir que les rendements décroissants viendraient principalement d'une confiscation des fruits du travail par une minorité, via le surtravail, ne suffit plus à expliquer l'énorme impasse technique et écologique dans laquelle nous nous trouvons aujourd'hui. Une confiscation des moyens de production ouvrant la voie à une démocratisation, c'est-à-dire une généralisation des techniques « les plus avancées » à l'échelle de l'humanité, ne produirait pas le communisme mais un appauvrissement quasi immédiat des ressources et une destruction générale des écosystèmes. L'utopie marxiste anticipait une augmentation indéfinie des forces productives - obtenue par un perfectionnement infini de la technique ${ }^{[10]}$, la question des «limites » s'en tenant souvent à la dénonciation, souvent fondée par ailleurs, des arguments malthusiens régulièrement maniés par les capitalistes contre les revendications ouvrières.

De ce fait, l'utopie de Marx ne comprend pas de théorie positive des besoins ${ }^{[1]}$ qui permettrait de savoir où arrêter la croissance de la consommation - et donc orienter l'évolution technique vers autre chose que le pouvoir d'achat maximum. «Mieux partager le gâteau ne suffit plus, c'est la recette qui doit être changée »: voilà le slogan qui est de plus en plus sur toutes les lèvres, et qui emmène avec lui toute la discussion sur les «nouvelles richesses » qu'il convient de défendre et non de s'en tenir au pouvoir d'achat ${ }^{[12]}$. Le cosmopolitisme de Marx est donc lui aussi en crise - et ce, indépendamment de l'effondrement de l'URSS dont les causes sont diverses.

\section{Le cosmopolitisme écologique}

Face à cette insuffisance des anciennes utopies émerge la critique écologiste. L'enjeu central est de faire durer « l'équilibre des écosystèmes », ordre décrit par l'écologie, c'est-à-dire d'éviter à la fois la «tragédie des communs ${ }^{[13]}$ » et le productivisme des États à économie planifiée. L'ouvrage qui trace le mieux les contours de l'utopie écologiste est sans doute le rapport Europe Soutenable ${ }^{[14]}$ (désormais nommé «RES»), réalisé en 1995 par le Wuppertal Institute pour les Amis de la Terre Europe. Rares sont les écrits militants aboutissant à une description d'une telle précision. 
Que propose le RES? Il se donne pour but de décrire une Europe qui aurait renoncé aux ressources épuisables et partagé à égalité les ressources renouvelables. La pierre angulaire du raisonnement est l'« espace environnemental », qui détermine deux bornes. La première est un maxima, obtenu en divisant le flux matériel maximum disponible par le nombre de bénéficiaires. Le rapport RES part de l'idée que les services écologiques se présentent sous la forme de « ressources » (matières premières ou « inputs ») et « d'éviers » (absorption des déchets ou « outputs »). L'équilibre entre les deux assure le maintien des flux, et donc la renouvelabilité des services: matière et énergie circulent de manière harmonieuse. À ce point d'équilibre correspond un « output » et un « input », un niveau maximal de prélèvement et de rejet. La seconde borne est un minima, déterminé par le concept de «besoin » au sens de Manfred Max-Neef, comme ce qui semble nécessaire à mener une vie normale dans une société donnée. Entre les deux peut librement s'exprimer la diversité culturelle des modes de vie, mais aussi la créativité technique, les manières les plus efficaces d'utiliser les ressources mélangeant « low-tech » (cuiseurs solaires, circuits d'alimentation courts, maisons bioclimatiques etc.) et high-tech (réfrigérateurs classe « $\mathrm{A}$ », etc.), un éclectisme revendiqué. Le respect de ces règles conduit à des réductions de 80 à $90 \%$ dans les prélèvements européens moyens. Dans le domaine du climat, par exemple, cela conduit à diviser par 5 ou 10 nos émissions actuelles. Dans le domaine de la pêche, cela conduirait à réduire fortement notre consommation de poisson. Et ainsi de suite.

La démarche du RES implique de donner aux ressources le statut de res communis, comme l'a montré notamment S. Chauvier ${ }^{[15]}$. Une res communis, en effet, est un bien dont toute personne a le droit de bénéficier et dont personne n'a le droit d'abuser ${ }^{[16]}$. Cela implique que les droits des personnes soient proportionnels, d'une part au nombre de personnes qui bénéficient de ce bien, et d'autre part à l'usage qui doit être fait de ce bien pour que sa qualité ne se détériore pas. Si une personne ou une collectivité jouissait d'un droit illimité d'appropriation, nous aurions affaire à une res nullius et non à une res communis. Le statut de res communis implique que les droits à bénéficier du bien soient assortis de limites, c'est-à-dire de devoirs. Ces devoirs sont définis par le nombre de personnes qui bénéficient du bien mais aussi par l'exigence de continuité dans la qualité du bien. S'il n'y a plus de bien, si les écosystèmes, etc., sont irrémédiablement détériorés, alors les droits n'ont plus de sens - nul ne prétendrait avoir des droits sur un «mal» commun.
[15] S. Chauvier, Justice et droit à l'échelle mondiale. Six études de philosophie cosmopolitique, coll. "Contextes ", Vrin, Paris, 2006, p. 61-87. Voir aussi B. Badie et M.-C. Smouts, Le retournement du monde. Sociologie de la scène internationale, $3^{\mathrm{e}}$ éd., Presses de Sciences Po \& Dalloz, Paris, 1999, et E. Ostrom, Governing the commons. The evolution of institutions for collective action, Cambridge Univ. Press, Cambridge, 1990. [16] S. Chauvier, op. cit. 
[17] J. Locke, Deuxième traité du gouvernement civil, Vrin, Paris, 1985 [1690], Chapitre V.

[18] Voir l'étude classique de K. Polanyi, La grande transformation. Aux origines politiques et économiques de notre temps, Gallimard, Paris, 1983 [1944].

[19] H. Grotius, Le droit de la guerre et de la paix, 1625 , chapitre XII relatif au « Tempérament par rapport à la dévastation et autres choses semblables ".

[20] P. Manent, Histoire intellectuelle du libéralisme, Calmann-Lévy, Paris, 1987.
Cherchant à protéger « la nature », les écologistes des Amis de la Terre cherchent donc en réalité à protéger des droits de l'humanité et, plus largement, les droits de tous les ayants droit à la res communis. L'enjeu est de protéger un ensemble de ressources qu'aucune personne ni aucun peuple particulier d'aucune époque particulière ne peut s'approprier, ne peut détruire, car toute personne, passée, présente ou à venir, y a droit. C'est là une interprétation très restrictive de la « clause lockéenne », qui correspond probablement davantage à l'esprit de son auteur ${ }^{[17]}$. On rejoint aussi des idées plus anciennes telles que les «communaux » du Moyen Âge, qui furent démantelés par les « enclosures » et la propriété privée au sens marchand du terme ${ }^{[18]}$. Grotius enjoignait ainsi de limiter la dévastation produite par la guerre en affirmant qu'il fallait protéger les arbres fruitiers, de même que les laboureurs et leurs maisons ${ }^{[19]}$. Les peuples passent, le laboureur reste, il change simplement de nationalité.

Cette approche ambitionne clairement de limiter la sphère de l'échange marchand, qu'il soit régulé ou auto-organisé. La res communis ne peut être cédée. Elle ne peut pas non plus être simplement «nationalisée », si par là on entend une appropriation simplement nationale. Ce qui est désigné comme « naturel » ici et dont il faut préserver l'intégrité, c'est l'ordre que toute personne doit s'efforcer de trouver dans le milieu, par-delà la succession des sociétés, des peuples et des familles, afin que des sociétés, des peuples et des familles puissent continuer d'exister, de trouver des «biens » dont elles peuvent tirer leur mode de vie propre. Cela comprend un certain nombre de pratiques comme la protection des sources, de la fertilité des terres, etc. On ne peut pas imposer aux personnes d'assurer la permanence de l'État, du peuple ou de la famille dans laquelle elles sont nées, mais par contre elles ont le devoir de laisser à autrui les moyens d'assurer sa subsistance par son travail. Ces moyens sont des éléments matériels mais aussi l'ensemble des savoirs nécessaires pour en maintenir la qualité.

À l'inverse, les théories de la «durabilité faible » issues des utopies technomarchandes considèrent qu'il n'existe d'autre res communis que les lois du marché, qui doivent être préservées envers et contre tout, en regard desquelles ressources et éviers sont avant tout destinées à être exploitées jusqu'à ce que la loi des rendements décroissants commande d'en abandonner l'extraction pour se déplacer et utiliser de nouveaux gisements. Le riche est celui qui hérite de la plus grosse « fortune », autrement dit celui qui sait provoquer sa propre richesse matérielle à force de ruse exercée principalement contre la nature ${ }^{[20]}$. On voit toute la différence entre les deux utopies. Ce contraste montre aussi à quel point la critique du luxe et du profit est consubstantielle à l'approche 
écologiste, ainsi que l'illustrent les dernières évolutions au sein de la mouvance écologiste ${ }^{[21]}$. Néanmoins, l'utopie écologiste ne peut se contenter d'être « anticapitaliste » au sens marxiste du terme, car cela reviendrait à risquer de reconduire le productivisme sous une forme étatiste.

Si l'espace environnemental est la pierre de touche de l'utopie écologique, alors l'institution de la res communis est bien au cœur de la démarche. L'espace environnemental est la conséquence de l'institution de la res communis. Nous nommons «utopie écologiste » un monde dans lequel les res communis seraient effectivement respectées.

À l'échelle mondiale, l'utopie décrite jusqu'ici est portée par ce qu'on appelle communément « le mouvement écologiste ». Celui-ci a été récemment décrit par Jean Jacob ${ }^{[22]}$. Les auteurs sont nombreux et bien identifiés. Ils dirigent souvent des ONG ou des think-tanks puissants et écoutés ${ }^{[23]}$. Bien que Jean Jacob en oublie quelques-uns dans son ouvrage, notamment l'Indien Anil Agarwal, il montre bien la consistance de ce courant, en termes d'actions communes et de citations croisées. Les personnes mentionnées se rencontrent souvent, écrivent des livres en commun, participent aux mêmes événements, sont dans les comités éditoriaux les uns des autres. Il montre que l'IFG joue un rôle central dans la structuration actuelle du mouvement mais que les racines sont bien plus anciennes. Elles remontent aux années 1980, voire aux années 1970. Si l'IFG a joué un rôle central pour catalyser les manifestations de Seattle contre l'OMC en 1999, le premier contresommet alternatif remonte à 1984, à Londres, avec l'organisation du TOES (The Other Economic Summit). En France, c'est Ecoropa (Ecological Europa) qui est le premier relais de ce courant, en 1976. En 1996, sa directrice, Agnès Bertrand, crée l'Observatoire de la mondialisation dont la présidence est confiée à Susan George. Elle crée la Coordination pour le contrôle citoyen de l'OMC (CCCMOC) qui regroupe 95 associations dont les revendications sont plus radicales que celles d'Attac. Corinne Lepage a représenté Ecoropa en 1994 pour faire accepter le principe de précaution au Conseil d'État en 1998. Ce courant a des ramifications avérées en France. S'il est passé relativement inaperçu jusqu'ici dans notre pays, c'est peut-être parce que les yeux sont rivés sur Attac, qui a été un mouvement très fort en France ces dernières années. La rivalité entre Attac et ce mouvement n'est pas neuve. Quand Bernard Cassen écrit Tout a commencé à Porto Alegre ${ }^{[24]}$, il ne dit rien de ce mouvement qui a toujours été présent dans les forums sociaux mondiaux. En France, ce sont les mouvements proches de la décroissance qui semblent désormais porter des orientations proches de celles de l'IFG: Serge Latouche et La Ligne d'Horizon, les revues
[21] J. Lindgaard, « N. Hulot: "Le capitalisme est obsolète" ", Mediapart, 14 septembre 2008.

[22] J. Jacob,

L'antimondialisation. Aspects méconnus d'une nébuleuse,

Berg International Editeurs,

Paris, 2006.

[23] Parmi les nombreuses personnalités influentes dans ce mouvement, on rencontre par exemple l'Indienne Vandana Shiva qui dirige Navdanya (www.navdanya. org), Research Foundation for science, Technology and Ecology (RFSTE), une organisation qui défend la biodiversité et promeut l'agriculture biologique par la dissémination de semences sans OGM ni brevets. On trouve aussi l'Étatsunien Jeremy Rifkin qui préside la Fondation on Economic Trends et est auteur de nombreux livres à succès, Lori Wallach qui est à la tête de Public Citizen (www. citizen.org), une ONG fondée en 1971 pour défendre les consommateurs et qui dispose aujourd'hui d'un budget de 11 millions de dollars, le Malais Martin Khor qui est le fondateur du Third World Network (www. twnside.org.sg) présent en Afrique, en Asie du SudEst, en Amérique latine et à Genève, ou encore Jerry Mander, actif dans la Fondation pour l'écologie profonde (www.deepecology. org) et actuel directeur de I'International Forum on Globalisation (IFG).

[24] B. Cassen, Tout a commencé à Porto Alegre... Mille forums sociaux, Mille et une nuits, Paris, 2003. 
L'Écologiste, Silence et Casseurs de Pub, Edgar Morin, les ONG écologistes, etc.

\section{Les points en débat}

Le rapport Europe soutenable n'est pas très bavard sur trois points qui sont pourtant très importants : la Sittlichkeit, ou éthique de la société civile, la technique et l'État. Voyons ce qu'ils deviennent si l'utopie écologiste est menée jusqu'au bout.

Le premier point est le plus aisé à saisir, car il est régulièrement au centre des débats : c'est la place du « capitalisme », la chrématistique au sens d'Aristote, la libre entreprise à but lucratif par rapport aux autres éthiques en jeu pour faire société - économie sociale, étatisme, etc. On sait que cette place a varié au cours de l'histoire. L'émergence de nouveaux indicateurs comme l'empreinte écologique et de nouveaux comportements comme les consom'acteurs ou la Responsabilité sociétale des entreprises (RSE), montre un souci de limiter cette sphère du profit. Mais les différents courants écologistes ne sont pas d'accord entre eux sur la place occupée par le capitalisme ${ }^{[25]}$. Certains lui laissent encore une place conséquente tandis que d'autres souhaitent l'en expurger totalement, sans toujours en tirer toutes les conséquences ni même utiliser la même définition de «capitalisme ». Quelle que soit l'issue du débat, les écologistes s'accordent sur le fait que l'histoire joue désormais contre l'éthique capitaliste. Quand on examine les scénarios de long terme, on ne voit guère comment elle pourrait persister à la place qu'elle a réussi à obtenir, ce qui implique que les sociétés industrialisées entrent dans une décroissance dont l'utopie écologiste souhaite qu'elle soit soutenable et équitable. La décroissance est ici envisagée comme transition vers un nouveau mode de vie qui serait « soutenable» dans tous les sens du terme. Mais à quelle vitesse et sous l'influence de quels facteurs la décroissance aura-t-elle lieu ? Sera-t-elle choisie ou subie? C'est un sujet de débats.

Les mouvements écologistes ne s'accordent pas non plus sur la nature des activités du «tiers secteur » qui occuperait la place laissée vacante. Par quoi remplacer l'éthique économique? L'« entrepreneuriat de cause »? L'activité démocratique? Les propositions vont dans le sens de renforcement d'éthiques préexistantes, issues notamment du don maussien, mais elles ajoutent aussi un élément original. Le souci d'équilibrer ses rapports avec la nature n'est en effet pas sans rappeler

[25] Parmi les protagonistes du débat, citons A. Gorz,

J. Zin... l'esprit du don: donner, recevoir et rendre - non pas directement aux êtres humains, mais à la nature. Donner, recevoir et rendre au monde 
dans son ensemble ${ }^{[26]}$. Celui qui rend à la nature permet aux échanges avec la nature de se perpétuer sous une forme équitable, et donc équilibrée. L'intégrité des « ressources » et des « éviers » est préservée. Le point qui est peu développé dans les propositions écologistes est de savoir dans quelle mesure cet acte de respect de la nature devrait aussi être à l'origine d'un respect des êtres humains, au motif que donner à la nature c'est indirectement contribuer à l'équilibre de sa communauté. Celle-ci ne devrait-elle pas le reconnaître? C'est en tout cas le sens de « l'environnementalisme des pauvres » dont parle J. Martinez Alier ${ }^{[27]}$. C'est par exemple les peuples autochtones d'Équateur qui demandent une rémunération pour ne pas extraire le pétrole qui se trouve dans le sol de l'une de ses réserves biologiques, ou Accion Ecologica qui réclame aux pays industrialisés la reconnaissance d'une «dette écologique ${ }^{[28]}$ », ou encore Anil Agarwal expliquant que les pauvres vont sauver le monde ${ }^{[29]}$. Celui qui oblige n'est plus celui qui donne matériellement parlant, mais celui qui se prive, du point de vue économique et matériel, au profit de la plénitude du monde. Les valeurs sont inversées: le riche devient le pauvre, celui qui est incapable de se relier au monde sans utiliser des masses d'objets encombrants et aliénants. L'autonomie économique, à ne pas confondre avec l'ancien ascétisme religieux, redevient d'actualité sous la forme de la «simplicité volontaire » ou de la « décroissance ». La composition sociologique des écologistes des pays industrialisés explique peut-être les difficultés qu'ils ont à aller jusqu'au bout de cette logique, pourtant fondatrice de leur combat, qui indique que politiser la question du luxe est la seule voie pour élargir leur audience vers les classes populaires, qui ont pour le moment délaissé leurs rangs.

Un second point est le statut de l'évolution technique et l'avenir du high-tech. Nous avons vu précédemment que l'impact d'une personne sur les écosystèmes est dépendant des techniques qui sont mises en œuvre, mais à leur tour ces dernières sont dépendantes de l'étendue de la division du travail mise en œuvre. Le rapport Europe soutenable n'a guère thématisé cette question mais un penseur important de l'écologie politique, Ivan Illich ${ }^{[30]}$, en a montré toutes les implications. Illich montre que les techniques les plus économes et les mieux appropriables sont souvent celles qui ne font pas appel à une division étendue du travail, car cette division exige transport, énergie et délégation à des entités qui deviennent lointaines et étrangères, engendrant de l'hétéronomie. Un raccourcissement de ces « détours de production » démesurés est nécessaire: telle est la conclusion de la plupart des comparatifs en termes d'« analyse de cycle de vie » (impact écologique global). C'est assez facile à comprendre et même à mettre en œuvre dans cer-
[26] F. Flipo, « Essai sur le don de la nature ", Entropia, $n^{\circ} 5$, p. ??-??.

[27] J. Martinez Alier, The environmentalism of the poor, Edward Elgar, Northampton, 2002. [28] http://fr.wikipedia.org/ wiki/Dette_écologique. [29] A. Agarwal, «Adressing the challenge of climate change: How poor nations can help to save the world ", texte publié sur le site de J.-M. Jancovici et daté de 2001: http://www.manicore. com.

[30] I. Illich, CEuvres complètes, Fayard, Paris, 2003. 
[31] Voir l'excellente synthèse rédigée par S. Fernandez, Low-tech, la deuxième voie technologique, 2002. http://www.econovateur. com/rubriques/anticiper/ voir011202.shtml.

[32] E. F. Schumacher, Small is beautiful. Une société à la mesure de l'homme, Seuil, Paris, 1978 [1973].

[33] Voir A. Gras et sa critique de l'évolutionnisme de Leroi-Gourhan: La fragilité de la puissance, Fayard, Paris, 2003, et Le choix du feu. Aux origines de la crise climatique, Fayard, Paris, 2007.

[34] Y. Coppens, L'histoire de I'homme, Odile Jacob, Paris,

2008.

[35] On citera encore une fois l'ouvrage de Luc Ferry, Le nouvel ordre écologique,

Grasset, Paris, 1992, et

de ses critiques, comme

exemplaire des tensions indiquant en creux le lieu des débats.

[36] B. Latour, Politiques de la nature, La Découverte, Paris, 2004. tains domaines comme l'alimentation - d'où les exemples toujours cités des Associations pour le maintien de l'agriculture paysanne (AMAP), des vacances en vélo, etc. Le respect des res communis conduit à des voies «low-tech », comme l'a remarqué Sauveur Fernandez ${ }^{[31]}$, à la suite de F. Schumacher ${ }^{[32]}$. Mais il y a des domaines plus difficiles et pour lesquels il n'existe pas de consensus fort au sein des mouvements écologistes. Pouvons-nous renoncer à nos machines IRM, ou aux ordinateurs? Les réponses ne sont pas simples, on l'imagine, d'où les âpres batailles autour des « choix technologiques ».

Les batailles sont d'autant plus rudes que la question de l'évolution technique implique, dans nos sociétés, un enjeu anthropologique. Bien des contemporains estiment en effet que les « progrès » réalisés dans la division du travail sont un trait constitutif de l'histoire naturelle de l'humanité ${ }^{[33]}$ - rien moins! Pour Yves Coppens, par exemple, l'histoire de la culture et de ses progrès semble s'identifier avec la multiplication des objets techniques ${ }^{[34]}$. Dès lors, tenter d'infléchir ce « destin » qui nous mène vers une maîtrise de plus en plus grande de « la nature », au moyen d'outils toujours plus perfectionnés, c'est risquer de renoncer à notre humanité, c'est être suspecté de vouloir « revenir à l'âge de pierre », de vouloir renoncer à « l'arrachement à la nature » qui serait constitutif de notre humanité. L'évolution technique est loin d'être une simple thèse académique sur l'histoire de l'humanité ; c'est un pan entier de notre cosmologie, ce qui explique que les débats entre « technophiles » et « technophobes » prennent rapidement la forme d'un débat « pour » ou « contre » l'homme lui-même ${ }^{[35]}$. On comprendra d'autant mieux la difficulté des débats si l'on se rend compte que si c'est effectivement la nature de l'homme qui est en question, si l'enjeu est réellement de nature anthropologique, ontologique, alors cela signifie aussi que nous entrons sur le terrain des explications religieuses, car les religions ont aussi des thèses à fournir dans ce domaine dont Kant avait estimé qu'il relevait de la métaphysique et dont il avait essayé de borner la violence.

Dès lors l'écologie n'est pas un discours qui vise à faire entrer la nature en politique, comme le suggère Bruno Latour ${ }^{[36]}$, mais un discours qui cherche à changer l'interprétation dominante de la nature - à savoir, l'évolutionnisme technique, l'idée selon laquelle l'être humain serait avant tout un Homo Faber destiné par nature à accrôitre sans cesse la finesse de ses outils par division du travail afin de « dominer la nature ». L'écologie introduit une conception de la nature qui se trouve en concurrence avec l'ancienne interprétation. Ce n'est pas un petit déplacement : c'est un programme (cosmo)politique complètement différent de ceux qui l'ont précédé. 
Le devenir de l'État pose un problème encore plus conséquent. Le rapport Europe soutenable ne s'y intéresse guère, sinon sous la forme d'un législateur abstrait dont la matérialité et l'organisation ne sont pas interrogées. Que devient l'État avec l'institution des res communis? À quelle échelle de gouvernance se manifeste-t-il? C'est ce qu'il faudrait savoir.

Soit, comme le suggère $\mathrm{S}$. Chauvier en s'appuyant sur Locke ${ }^{[37]}$, l'État conçu comme ayant pour but l'eunomia des personnes, n'existant que pour augmenter leur liberté. L'État rend certains services: éducation, justice, etc. Ces services, tout comme les biens (routes, etc.), sont le fruit de « détours de production ». Du point de vue de l'état des res communis, ce qui frappe, d'emblée, est le poids énorme des institutions étatiques dans les pays industrialisés. L'État central et ses agences, en bref l'ensemble des structures publiques et parapubliques contrôlent de près ou de loin près de $50 \%$ des échanges économiques. Le RES n'évalue pas le poids écologique de l'État mais un tel poids économique doit se doubler d'un poids écologique conséquent, quand bien même ne fournirait-il que des « services » réputés « immatériels ». J. Gadrey a montré les limites de cette conception ${ }^{[38]}$, les services rendus par l'État doivent donc être évalués dans le même cadre.

Si l'on applique les principes illichiens à l'État, alors cela implique, dans le domaine du gouvernement, une relocalisation. D'ailleurs les élus écologistes insistent largement sur le "principe de subsidiarité », qu'ils entendent généralement comme le fait de chercher à résoudre les problèmes au plus près des citoyens ${ }^{[39]}$, reprenant ainsi l'argument de Rousseau, ou encore leur soutien aux identités régionales. En ce sens, le cosmopolitisme écologiste ne repose ni sur la mobilité illimitée ni sur l'intégration mondiale dans une division du travail ayant la production maximale pour finalité mais sur la juste séparation. Ici, nous sommes solidaires parce que nous sommes tous respectueux des droits de nos voisins, et non parce que nous coopérons dans le but de produire des biens et des services. C'est la seule manière réaliste de produire ou de protéger des res communis susceptibles de donner une place à chaque personne sur Terre. Les Terriens associés vivraient non pas dans un seul État mais dans une fédération de régions autonomes sur le plan économique et vivant en bonne intelligence. Néanmoins, cela pose un certain nombre de problèmes spécifiques qui ne sont pas abordés dans le RES.

Réduire l'État ne revient-il pas à se dépouiller et à s'affaiblir devant les nouveaux empires, qui ne rêvent que d'étendre leur contrôle sur des territoires de plus en plus vastes? On pourra répondre que l'utopie cosmopolitique vise justement à instaurer des limites à la violence,
[37] S. Chauvier, op. cit., p. 61-87.

[38] J. Gadrey, Les services ne sont pas « la » solution à la crise écologique, Actes du premier colloque international sur la décroissance économique pour la soutenabilité écologique et l'équité sociale, Paris, 18 avril 2008, p. 47-52. http:// events.it-sudparis.eu/ degrowthconference/ themes/

[39] Exemple: http:// lesverts.fr/article.php3?id_ article $=401$ 
et qu'opposer la violence à la violence ne peut résoudre le problème. L'utopie écologiste s'appuie d'ailleurs sur les traditions de non-violence, notamment la désobéissance civile.

L'État ne va-t-il pas disparaître dans le très grand pluralisme d'États issus de l'utopie écologiste? N'allons-nous tout simplement pas retomber dans l'état de nature tant redouté ? Non, S. Chauvier a montré qu'un pluralisme d'États ne se confond pas nécessairement avec un libertarisme favorable à l'abolition totale des frontières ${ }^{[40]}$. L'État est le résultat d'une coopération profitable à toutes les personnes. Une mobilité illimitée ne tiendrait pas compte des désutilités créées par le départ ou l'arrivée impromptue des personnes. Dans les deux cas, les effets ne sont pas nuls et les personnes qui seraient lésées ont aussi des droits. S'il y a un devoir d'humanité à accueillir les personnes menacées dans leur pays, il n'y a aucun droit des étrangers à venir s'installer de manière durable, si cela a des conséquences sur la société habitant le territoire sur lequel ils envisagent de s'installer ${ }^{[41]}$. De même, les citoyens d'un État ne peuvent à loisir quitter cet État avec leurs biens, leur capital, si cela a pour conséquence de générer le désordre ou de réduire le bien-être de cette société. L'État, créé pour générer l'eunomia parmi ses membres, doit y veiller. Nulle conception holiste, par conséquent, ici, mais une approche individualiste authentiquement pluraliste. Les citoyens, comme l'État, peuvent aussi avoir un devoir d'ingérence, dans certains cas, par exemple si un autre État se retourne contre les citoyens qui l'ont créé et réduit de manière arbitraire ou despotique leur liberté.

Aller vers un très grand pluralisme d'États ne revient pas non plus à s'extirper de toute histoire et créer des communautés ex nihilo. Les autorités publiques existent déjà à toutes les échelles de gouvernance, de la municipalité de quelques habitants à l'ONU qui comprend la quasi-totalité des Terriens. Favoriser la relocalisation revient à traiter les problèmes à une échelle plus basse - «penser global, agir local »-, en faisant en sorte que les problèmes existants à une échelle supérieure disparaissent. La stratégie est donc de réduire les problèmes transfrontaliers et non de les augmenter, ce qui permet d'éviter de mettre en place une coûteuse administration dont l'objet est à chaque fois de gérer les problèmes créés, voire de créer les problèmes pour justifier son existence et son activité. L'utopie est cohérente avec elle-même, mais en contradiction avec une grande partie des dynamiques étatiques actuelles, qui sont au contraire en train d'étendre les bureaucraties globales, publiques ou privées...

Enfin, des États plus petits ne seraient-ils pas forcément « fermés » et renfermés? N'allons-nous pas perdre les acquis des grandes sociétés, notamment l'émancipation par rapport à la communauté et à la 
famille? N'est-ce pas la fin de la « société ouverte »? Le rapport RES n'est à nouveau pas très bavard, et les positions écologistes sont éclatées sur ce sujet. Néanmoins, des études récentes indiquent que rien ne prouve que le commerce marchand ait significativement réduit les occurrences de conflit entre nations ${ }^{[42]}$. Les personnes ont voyagé et échangé depuis toujours et n'ont pas attendu les échanges commerciaux pour cela. La petite communauté est valorisée dans toutes les utopies écologistes ${ }^{[43]}$. La famille est rarement prise pour cible de la critique en tant que telle, c'est plutôt une certaine forme de famille, autoritaire, qui est dénoncée.

\section{Conclusion : 1'utopie écologiste, un retour aux Lumières?}

L'institution des écosystèmes en res communis semble bien jouer le rôle de pierre de touche dans les revendications écologistes. Les conséquences les plus éloignées de ce projet font émerger un « village global » bien différent des anciennes utopies. Le RES actualise ainsi sans le savoir, ou tout du moins sans le mentionner, le principe universel du droit qui, chez Kant, fonde la justice: "Toute action est juste qui peut faire coexister la liberté de l'arbitre de chacun avec la liberté de tout autre selon une loi universelle, ou dont la maxime permet cette coexistence ${ }^{[44]} »$. La critique écologiste semble chercher à tirer les conséquences de ce principe dans le temps, mais aussi dans l'espace, en cherchant à inclure les «non-humains », au moyen des éthiques environnementales. Est-ce pour autant un retour aux Lumières qui nous est promis?

Ce n'est pas évident. Il y a en effet deux dérives possibles, qui vont croître au fur et à mesure que les risques pesant sur les res communis vont être perçus comme vitaux, c'est-à-dire au fur et à mesure que la substance de ces biens sera menacée et que ces conséquences se feront sentir dans la chair de celles et ceux qui en dépendent. Le premier est le renforcement de la tyrannie d'État, d'un État sur son peuple, d'un État sur les autres ou d'un ensemble de personnels des États sur l'ensemble de l'humanité. La réduction des circuits de production implique aussi la réduction des circuits de production de la loi, or les élites qui tirent leur pouvoir de leur monopole dans le règlement des conflits risquent de ne pas accepter de voir leur rôle remis en cause. Elles chercheront à justifier les privilèges liés à leur fonction.

Le second est que moins l'État est efficace, plus les personnes menacées risquent de chercher à sacraliser leurs droits sur la res com-
[42] P. Martin, T. Mayer et M. Thoening, La mondialisation est-elle un facteur de paix?, Éditions Rue D'Ulm, Paris, 2006. [43] Voir le site dédié à la transition (http://www. transitiontowns.org) et les illustrations de la ville idéale dans le journal $L a$ Décroissance.

[44] E. Kant, Métaphysique des mœurs, 1797, § C. 
munis, qui seront de plus en plus perçus comme un enjeu vital. Les ethnologues et les anthropologues ont ainsi montré le rôle des « bois sacrés » dans l'équilibre écologique d'une population. On se rappelle la déclaration du Chef Seattle, un grand classique chez les écologistes. De là sans doute le lien que Jean Jacob voit entre mouvements écologistes et mouvements religieux. Les grandes religions commencent d'ailleurs à comprendre que le « marché de l'angoisse », généré par les enjeux écologiques, n'avait pas été assez investi. 